\title{
Multifunctional Nanobiomaterials for Neural Interfaces
}

\author{
By Mohammad Reza Abidian* and David C. Martin
}

Neural electrodes are designed to interface with the nervous system and provide control signals for neural prostheses. However, robust and reliable chronic recording and stimulation remains a challenge for neural electrodes. Here, a novel method for the fabrication of soft, low impedance, high charge density, and controlled releasing nanobiomaterials that can be used for the surface modification of neural microelectrodes to stabilize the electrode/ tissue interface is reported. The fabrication process includes electrospinning of anti-inflammatory drug-incorporated biodegradable nanofibers, encapsulation of these nanofibers by an alginate hydrogel layer, followed by electrochemical polymerization of conducting polymers around the electrospun drug-loaded nanofibers to form nanotubes and within the alginate hydrogel scaffold to form cloud-like nanostructures. The threedimensional conducting polymer nanostructures significantly decrease the electrode impedance and increase the charge capacity density.

Dexamethasone release profiles show that the alginate hydrogel coating slows down the release of the drug, significantly reducing the burst effect. These multifunctional materials are expected to be of interest for a variety of electrode/tissue interfaces in biomedical devices.

\section{Introduction}

Recording and stimulating neural prostheses can improve our understanding of the organization and operation of the nervous system and may lead to improved prosthetic devices for tackling of some mankind's most debilitating disorders, including deafness, paralysis, blindness, epilepsy, and Parkinson's disease. Neural microelectrodes, such as microwires ${ }^{[1]}$ and microfabricated silicon electrodes, ${ }^{[2-4]}$ are capable of interfacing with the central and peripheral nervous system at the cellular level by transducing ionic biological signals to electronic signals. ${ }^{[5]} \mathrm{A}$ low impedance electrode/tissue interface is critically important to maintain signal quality for recording, as well as effective charge transfer for stimulation. However, neural microelectrodes often exhibit high initial electrode impedance because of their small

[*] Dr. M. R. Abidian

Department of Biomedical Engineering

The University of Michigan

1101 Beal Ave., Ann Arbor, MI 48109 (USA)

E-mail: mabidian@umich.edu

Prof. D. C. Martin

Departments of Biomedical Engineering,

Materials Science and Engineering, and

Macromolecular Science and Engineering

2644 CSE Building, Ann Arbor, MI 48109 (USA) surface area. ${ }^{[6,7]}$ Furthermore, encapsulation processes from the reactive cellular response progressively increase the impedance of electrode/tissue interface over the long term. ${ }^{[8-11]}$

Several studies have been conducted to explore strategies to reduce the initial impedance of electrode sites or to limit early and late reactive responses near the electrode/tissue interface. These strategies include: i) optimizing the size,$^{[10]}$ shape, ${ }^{[8,12]}$ tip geometry, ${ }^{[10]}$ texture, ${ }^{[10,13]}$ material substrate, ${ }^{[13-15]}$ and insertion procedures $^{[10,12,16,17]}$ to minimize the initial trauma to the brain tissue during implantation; ii) deposition of metals ${ }^{[18]}$ and metal oxides ${ }^{[19]}$ on the surface of electrode sites and applying bias voltage pulses $^{[20]}$ to decrease the initial impedance of the electrode; iii) bioactive coatings on the electrode using biomolecules, ${ }^{[21,22]}$ and the systemic or local delivery of antiinflammatory drugs ${ }^{[23,24]}$ to reduce the reactive tissue response and encapsulation process

Recently, conducting polymers such as poly(pyrrole) (PPy) and poly(3,4-ethylenedioxythiophene) (PEDOT) have been considered for biomedical applications because of their physical, electrical, and biocompatibility characteristics. ${ }^{[25,26]}$ Since, their responses to electrochemical or electronic signals can produce a change in conductivity and volume, conducting polymers are of interest for neural engineering applications such as neuronal cell signaling, ${ }^{[27]}$ neural interface, ${ }^{[28,29,30]}$ and controlled drug delivery. ${ }^{[1,32]}$ We have successfully demonstrated that PPy and PEDOT can be electrochemically polymerized on neural microelectrode sites in the form of films, ${ }^{[33]}$ interconnected microcavities, ${ }^{[34]}$ microfibrils, ${ }^{[35]}$ and nanotubes (NTs). ${ }^{[31]}$ These morphologies significantly increase the effective surface area of the electrode/tissue interface. As a result, the capacitance of the electrode site dramatically increases, creating a corresponding reduction in electrode site impedance. ${ }^{[30]}$ PEDOT exhibits higher conductivity and chemical stability than PPy in the oxidized state. ${ }^{[36-41]}$ We have demonstrated that PEDOT can be polymerized on neural electrodes and around electrospun nanofibers to form PEDOT nanotubes $^{[30]}$ (PEDOT NTs), and that these nanotubes can be used for the controlled release of anti-inflammatory drugs. ${ }^{[31]}$

Previous studies have described the importance of considering the dramatic differences in mechanical and electrical properties between an implanted microelectrode and the ionically active neural tissue. ${ }^{[9,13,28]}$ Hydrogels provide a soft mechanical buffer layer, but they move the electrode away from the target cells, and may release incorporated drugs too quickly. Conductive polymer

DOI: $10.1002 / \mathrm{adfm} .200801473$ 
coatings can significantly reduce electrode impedance, but they are relatively thin $(\sim 5 \mu \mathrm{m})$ and only provide a modest mechanical buffer effect. Drugs such as dexamethasone (DEX) can be incorporated into biodegradable matrices, but these polymers are insulators and would prevent the electrodes from functioning properly if used in a simple continuous coating. It is therefore of interest to find techniques that could create surfaces that are simultaneously mechanically soft, low impedance, and able to release drugs in a controlled fashion.

In this study, we describe the design and characterization of a multifunctional, hybrid nanostructured interface for neural microelectrodes that is soft, low impedance, has high charge density, and is capable of controlled drug release. Our design includes: i) biodegradable electrospun nanofibers for the controlled release of drugs and to provide a scaffold for the formation of conducting polymer nanotubes and ii) hydrogel layers for the sustained release of drugs, and to provide a scaffold for formation of nanostructured cloud-like conducting polymer. These hydrogel coatings provide a mechanical buffer layer between the hard silicon-based probe and the soft brain tissue, a scaffold for growing the conducting polymer within the hydrogel matrix, and a diffusion barrier for controlling drug release. Since high electrical conductivity is one of the most important parameters for achieving stable communication with neurons, and the electrospun polymer layer used in this experiment was an electrically nonconductive material, it was necessary to enhance the conductivity of the nanofibers using conducting polymers. In order to enhance the electrical properties of the electrode sites, PEDOT was electrochemically polymerized on the electrode sites, around the nanofibers and eventually inside the alginate hydrogel matrix. Figure 1 shows the fabrication process that includes electrospinning of drug-loaded biodegradable nanofibers (Fig. 1B), hydrogel coating of the neural electrode using a dipping method (Fig. 1C), and finally electrochemical polymerization of conducting polymer around the electrospun nanofibers within the hydrogel scaffold (Fig. 1D). We believe that the hydrogel layer stabilizes the release of DEX due to hydrolytic degradation of the electrospun fibers. This controlled release should reduce the risk of exposure to high systemic doses of DEX that are associated with serious side effects such as diabetes, hemorrhagic ulcer, skin atrophy, and osteoporosis. ${ }^{[42,43]}$

\section{Results and Discussion}

\subsection{Electrospinning of Dexamethasone-Loaded Nanofibers on the Neural Electrode}

Among bioresorbable polymers, poly( $\alpha$-hydroxy acids) such as poly(L-lactic acid) (PLLA) and poly(lactic-co-glycolic acid) (PLGA) have been extensively used as drug carriers ${ }^{[44-46]}$ and scaffolds for tissue engineering ${ }^{[47-49]}$ because of their tailorable biodegradability and good biocompatibility. ${ }^{[50]}$ These polymers degrade into nontoxic products and have received United States Food and Drug Administration approval for medical devices. ${ }^{[51]}$ As shown in Figure 2B-D, DEX-incorporated electrospun nanofibers were prepared and collected on the neural microelectrodes. Figure 3 shows scanning electron micrographs (SEM) of DEX-

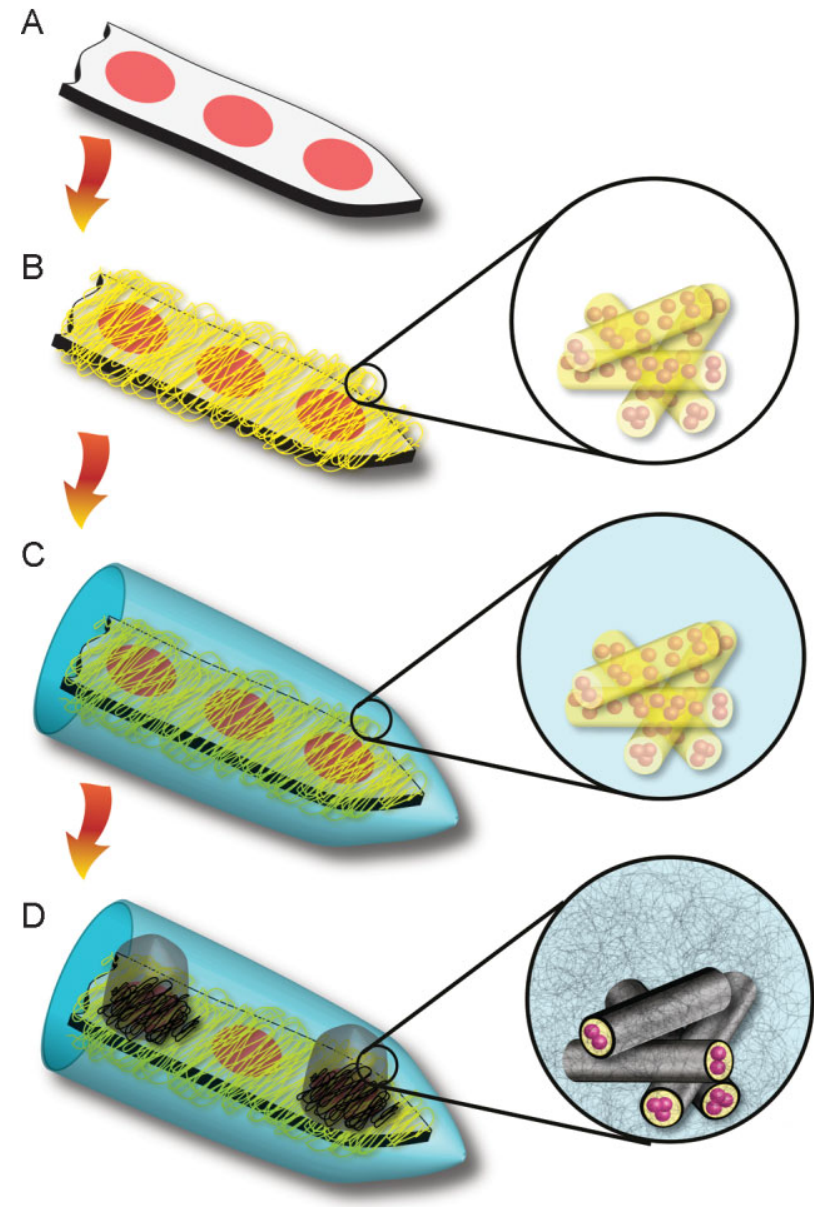

Figure 1. Schematic diagram of the fabrication process for multifunctional polymer coatings on the neural microelectrodes: A) uncoated microelectrode, B) electrospinning of DEX-loaded biodegradable nanofibers, C) alginate hydrogel coating, D) electrochemical polymerization of PEDOT on the electrode sites, around the DEX-loaded electrospun biodegradable nanofibers, and within the hydrogel scaffold.

loaded poly(L, D-lactic-co-glycolic acid) with composition ratio of LA/GA 75:25 (PLDL75G25A)/DEX (ratio 10:1), poly(L, D-lactic acid) (PLDLA)/DEX (ratio 10:1), and PLLA/DEX (ratio 4:1). PLLA has higher molecular weight than PLDL75G25A and PLDLA, therefore, the solubility of PLLA in chloroform is less than PLDL75G25A and PLDLA. DEX was completely dissolved in the chloroform with the concentration of $15.6 \mathrm{mg} \mathrm{mL}^{-1}$ for PLLA/ DEX solution (ratio 4:1) and $22.5 \mathrm{mg} \mathrm{mL}^{-1}$ for PLDL75G25A/ DEX, and PLDLA/DEX solution (ratio 10:1), which is in the range of maximum solubility of DEX in chloroform (maximum concentration of $25 \mathrm{mg} \mathrm{mL}^{-1}$ ). The diameters of the DEX-loaded nanofibers ranged from 40 to $150 \mathrm{~nm}$ with the majority between 55 and $96 \mathrm{~nm}$ (Fig. 3). In contrast to PLDLA/DEX and PLDL75G25A/DEX, the surface texture of the PLLA/DEX fibers had a well-defined porous morphology (Fig. 3E and F). ${ }^{[31,52]}$ Since, PLDLA and PLDL75G25A are amorphous polymers and did not exhibit this porous surface morphology when processed from the same solvent, it may be that the crystallization of PLLA caused this surface structure. As shown in optical micrographs of 

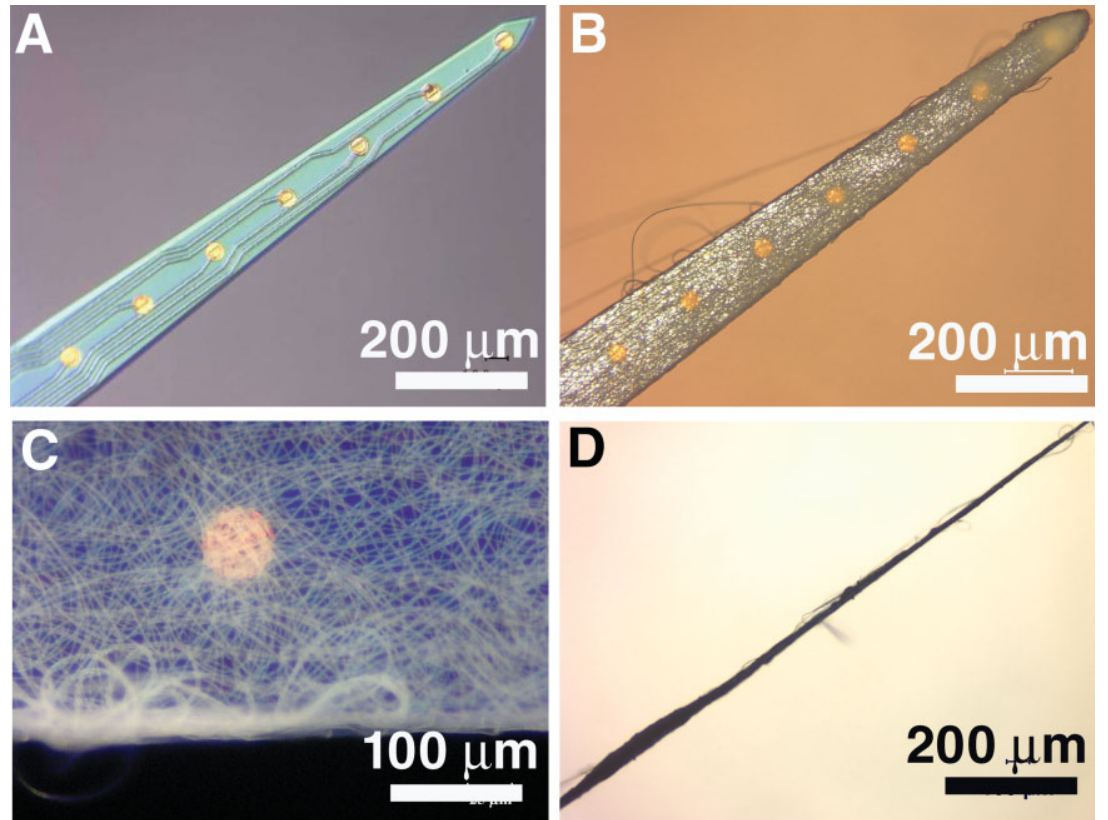

Figure 2. Optical micrographs of A) silicon-based eight-channel recording gold sites with surface area of $1250 \mu \mathrm{m}^{2}$, B) top view of electrospun DEX-loaded biodegradable nanofibers on the surface of silicon electrode, C) higher magnification of (B) showing single gold site and random distribution of nanofibers on the surface of neural electrode, D) side view of (B) showing the thickness of nanofibers on the neural electrode.
Figure 2, the nanofibers were distributed randomly in a mat on the surface of the probe and covered the entire shank of the neural probe. The thickness of the resulting fiber mat was $24 \pm 8 \mu \mathrm{m}$ on each side of electrode for 1 min of electrospinning with the DEX-polymer solution.

When the concentration of DEX in the solutions was increased beyond the solubility limit $\left(25 \mathrm{mg} \mathrm{mL}^{-1}\right)$, the excess DEX particles aggregated together and were covered by layers of polymers along the fiber strings (Fig. 4). The size of the vesicles containing the DEX particles was in the range of $5-20 \mu \mathrm{m}$. Figure 4 shows SEM images overloaded DEX in PLDLA (PLDA/DEX, 8:1), PLDL75G25A (PLDL75G25A/DEX, 8:1), and PLLA (PLLA/ DEX, 2:1) with DEX concentration of 33.2, 33.2 , and $31.2 \mathrm{mg} \mathrm{mL}$ in chloroform, respectively.

\subsection{Alginate Coating of the Neural Electrode}

After electrospinning the drug-loaded biodegradable polymer nanofibers (PLDL75G25A/
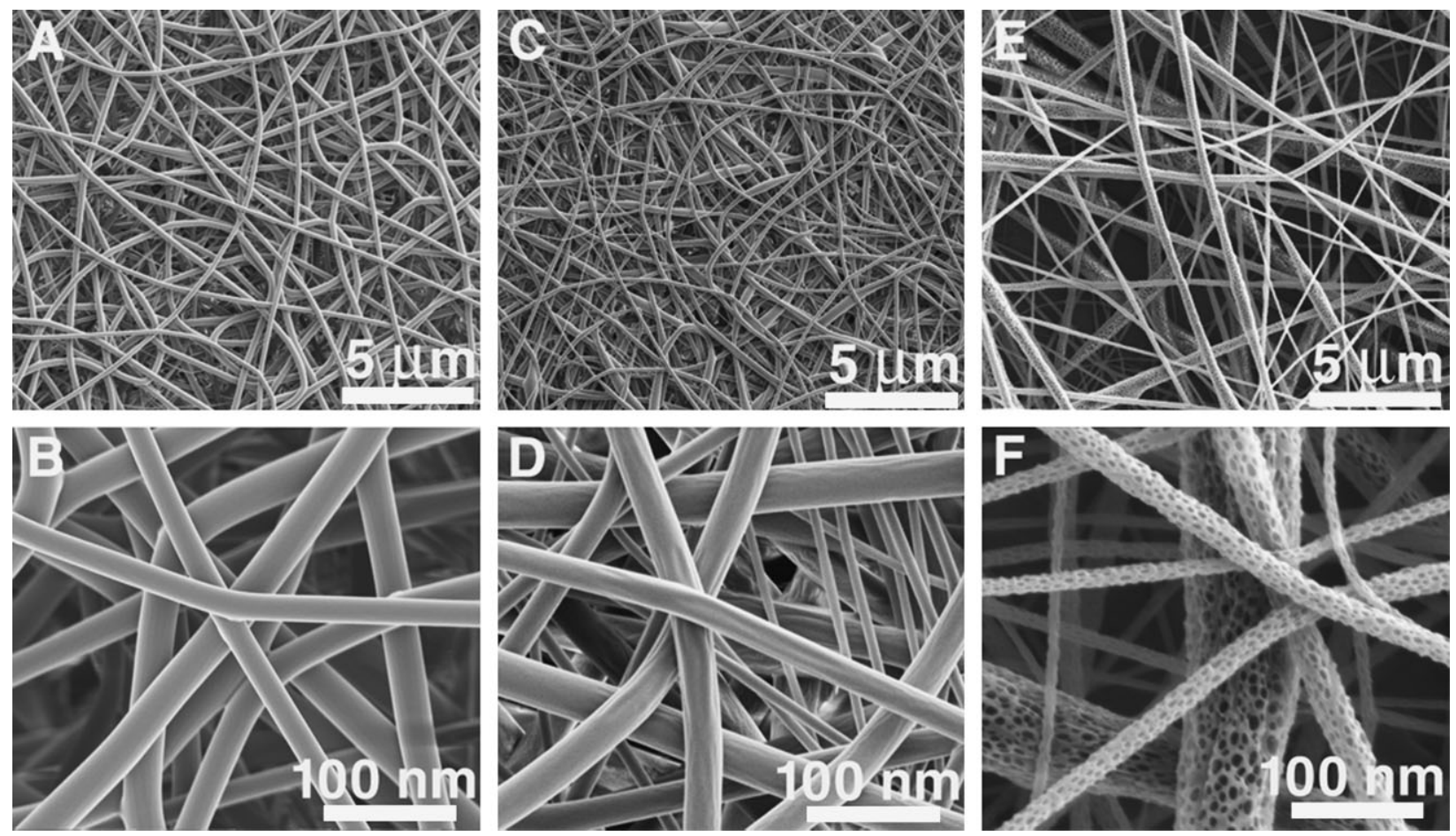

Figure 3. SEMs of DEX-loaded electrospun: $A$ ) and $B$ ) PLDL75C25A nanofibers (PLDL75G25A/DEX, 10:1), C) and D) PLDLA nanofibers (PLDLA/DEX, 10:1), E) and F) PLLA nanofibers (PLLA/DEX, 4:1). DEX was completely dissolved in the chloroform with the concentration of $15.6 \mathrm{mg} \mathrm{mL}^{-1}$ for PLLA/DEX solution and $22.5 \mathrm{mg} \mathrm{mL}^{-1}$ for PLDL75C25A/DEX and PLDLA/DEX solution which is in the range of solubility of DEX in chloroform (maximum concentration of $25 \mathrm{mg} \mathrm{mL}^{-1}$ ). The diameters of the DEX-loaded nanofibers ranged from 40 to $150 \mathrm{~nm}$ with the majority between 55 and $96 \mathrm{~nm}$. 

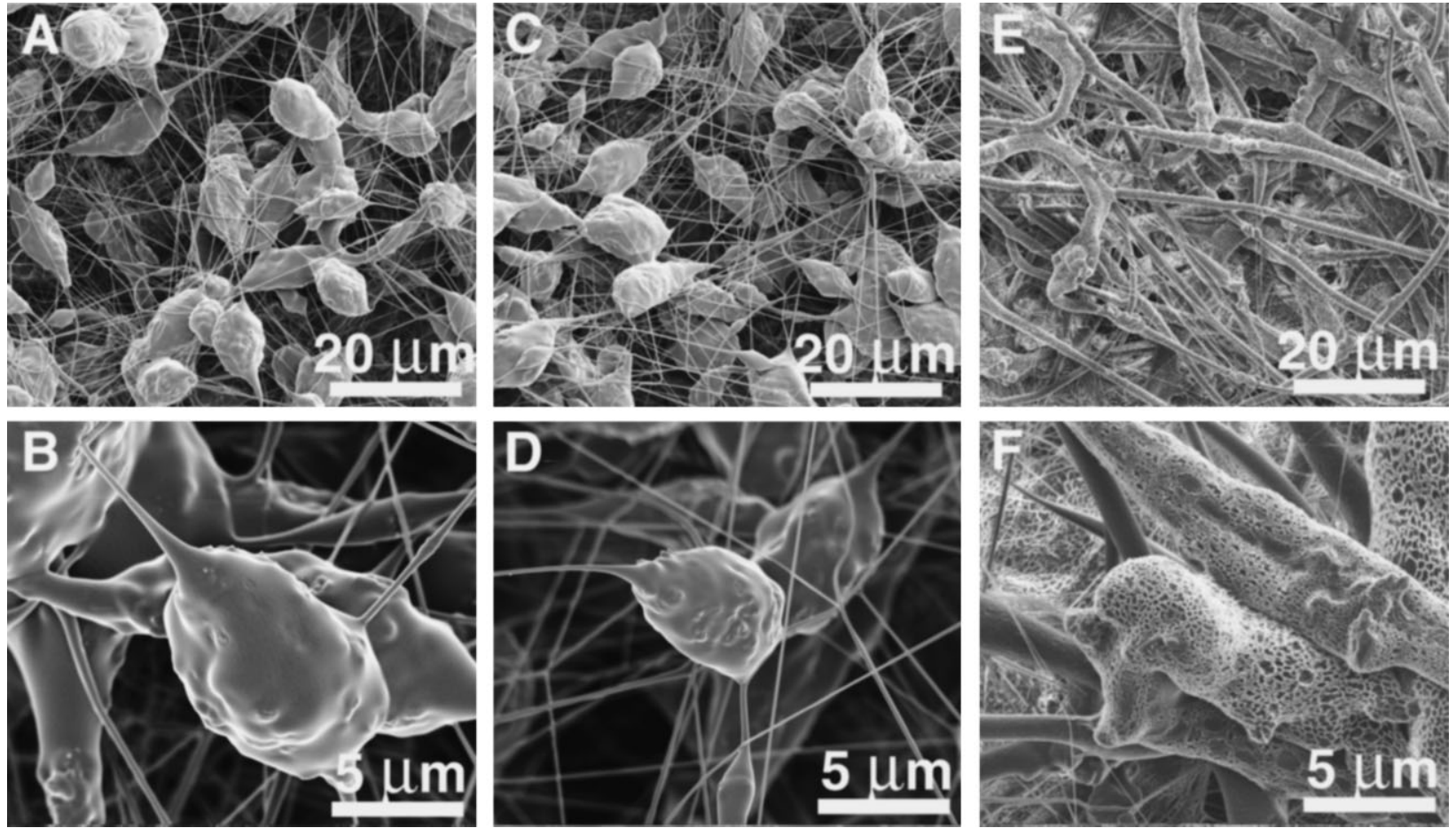

Figure 4. SEM of DEX-loaded electrospun $A$ ) and B): PLDL75G25A nanofibers (PLDL75G25A/DEX, 8:1); C) and D): PLDLA nanofibers (PLDLA/DEX, 8:1); E) and F): PLLA nanofibers (PLLA/DEX, 2:1). DEX was dissolved in the chloroform at a concentration of $31.2 \mathrm{mg} \mathrm{mL}^{-1}$ for PLLA/DEX solution and $33.2 \mathrm{mg}$ $\mathrm{mL}^{-1}$ for PLDL75C25A/DEX and PLDLA/DEX solution which is beyond the range of solubility of DEX in chloroform (maximum concentration of $25 \mathrm{mg}$ $\mathrm{mL}^{-1}$ ). The size of the vesicles containing the DEX particles was in the range of 5-20 $\mu \mathrm{m}$. The excess DEX particles aggregated together and were covered by layers of polymers along the fiber strings.

DEX), alginate was ionically crosslinked by $\mathrm{Ca}^{2+}$ ions using a dip coating method which covered the entire shank of neural microelectrodes. The thickness of hydrogel coating depends on the concentration of alginate polymer, $\mathrm{Ca}^{2+}$ ions in the solutions, as well as the number of dipping cycles. It was found that the lateral movement of the neural electrode and speed of dipping effects on the uniformity of the alginate coating around the shank of the probe. Figure $5 \mathrm{~A}-\mathrm{C}$ shows optical micrographs of the top and side views of alginate hydrogels in hydrated state that covered the entire shank of the neural probe. The overall thickness of hydrogel was $360 \pm 23 \mu \mathrm{m}$ in hydrated condition after 4 dipping cycles in $1 \% \mathrm{w} / \mathrm{w}$ alginate and $0.5 \mathrm{M} \mathrm{CaCl}_{2}$ solutions. However, this thickness decreased to $60 \pm 5 \mu \mathrm{m}$ in dehydrated (dried) situation, which is the total thickness of DEX-loaded PLDL75G25 nanofibers on both sides of the neural probe (Fig. 5D-F). To minimize the amount of damage during the implantation, ${ }^{[9]}$ coated neural electrodes should be inserted into the brain in the dehydrated state. Once the electrode is placed in the tissue, it will rehydrate from water naturally available in the tissue. ${ }^{[53]}$ It is therefore necessary to have conducting pathways through the gel so that it is possible to maintain communication with the cells that might be pushed from the electrode when the gel re-swells. Figure 5G and $\mathrm{H}$ shows SEM images of dehydrated alginate on the surface of a neural electrode that shows the electrospun nanofibers underneath the dried alginate membrane.

\subsection{Electrochemical Polymerization of PEDOT in Hydrogel Scaffolds}

PEDOT was electropolymerized on the $1250-\mu \mathrm{m}^{2}$ gold electrode sites that were coated by DEX-loaded PLDL75G25A electrospun nanofibers and alginate hydrogel. The total applied charge density during electrochemical deposition was $2.88 \mathrm{C} \mathrm{cm}^{-2}$ which caused the minimum impedance ${ }^{[30,31]}$ (applied current $20 \mathrm{nA}$ for $1800 \mathrm{~s}$ ). Figure 6A shows top view of the deposited PEDOT on the electrode site (black color). The side view optical microscope image (Fig. 6B) revealed that PEDOT was grown vertically from the conductive site, around the electrospun nanofibers, ${ }^{[31]}$ and gradually expanded throughout the hydrogel structure with a cloud-like morphology. Visual inspection showed that as electropolymerization time increased, PEDOT content also increased. For electrochemical polymerizations longer than 30 min, the black PEDOT phase had vertically permeated up to the surface of hydrogel. The PEDOT grows around the filaments of the alginate gel, preserving the open structure and pathways for transport through the aqueous phase. ${ }^{[54]}$ Figure $6 \mathrm{C}$ and D shows SEM images of PEDOT NTs that were left on the electrode site after dissolving away the alginate coating in deionized water and PLDL75G25A nanofibers in chloroform. These images show the formation of PEDOT around the DEX-loaded PLDL7525GA electrospun nanofibers. ${ }^{[30,31]}$ 

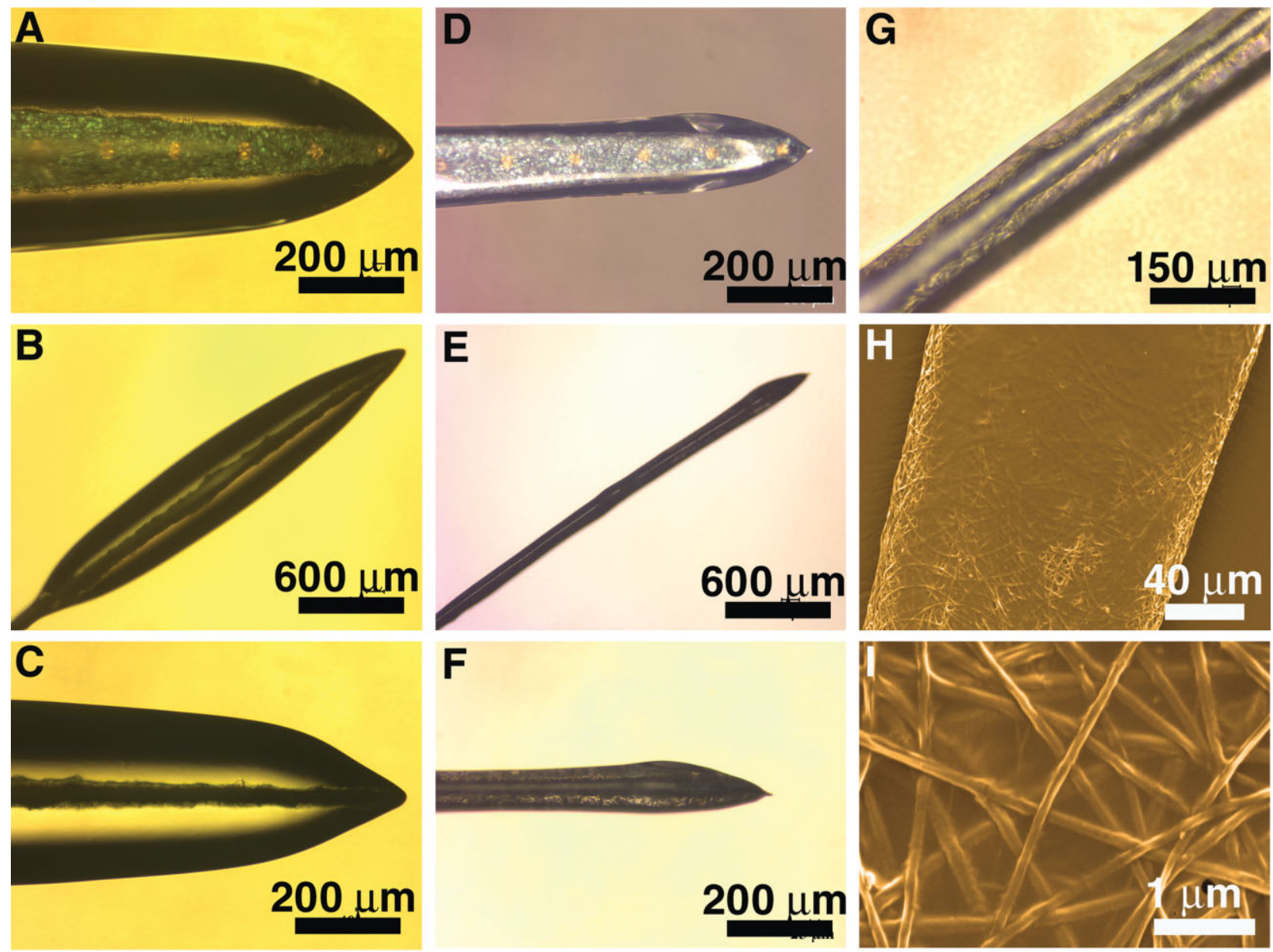

Figure 5. Optical micrographs of A) top view of neural electrode after coating with DEX-loaded electrospun nanofibers and alginate hydrogel in hydrated state, B) side view of (A) which shows entire electrode, C) side view of (A) which is higher magnification of (B), D) top view of neural electrode after coating with DEX-loaded electrospun nanofibers and alginate hydrogel in dehydrated state, E) side view of (D) which shows entire electrode after dehydration of alginate, F) side view of (D) which is higher magnification of in dehydration of alginate (E), G) higher magnification of (F) which shows thickness of alginate hydrogel after dehydration and electrospun nanofibers inside of hydrogel on top of electrode, $\mathrm{H}$ ) false-colored SEM images of neural electrode after coating with DEX-loaded electrospun nanofibers and alginate hydrogel in dehydrated state of alginate, I) false-colored higher magnification of $(\mathrm{H})$.

\subsection{Electrochemical Impedance Spectroscopy (EIS)}

EIS was used to explore the conductivity of the polymer coatings over a frequency range $1-10^{5} \mathrm{~Hz}$ for PEDOT NTs, cloudy PEDOT inside the alginate hydrogel (HG + PEDOT), and PEDOT deposition around the electrospun nanofibers, and within the alginate hydrogel (PEDOT NTs + HG + PEDOT) (Fig. 7G). The impedances at $1 \mathrm{kHz}$ are particularly important because they correspond to the characteristic frequency of neuronal action potentials. ${ }^{[5]}$ Figure $7 \mathrm{G}$ shows EIS of the electrode site and the change in impedance as a function of different coatings. The electrode's impedance across all frequencies was moderately increased by the layer of nonconductive DEX-loaded PLDL75G25A nanofibers (Fig. 7B; PLGA NFs). This impedance increased slightly after coating with conductive hydrogel (Fig. 7C; PLGA NFs + HG). After PEDOT deposition around the DEXloaded PLGA nanofibers and within the alginate hydrogel (Fig. 7F; PEDOT NTs + HG + PEDOT), the impedance at $1 \mathrm{kHz}$ decreased by about two orders of magnitude from the unmodified electrode. We also compared the impedance of PEDOT $\mathrm{NTs}+\mathrm{HG}+\mathrm{PEDOT}$ with PEDOT NTs (Fig. 7E; PEDOT NTs without alginate hydrogel) and cloudy PEDOT (Fig. 7D; $\mathrm{HG}+$ PEDOT without PEDOT NTs). As shown in Figure 7G, the impedance of PEDOT NTs + HG + PEDOT is less than the PEDOT NTs, which are in turn less than HG + PEDOT. Although the impedance of PEDOT NTs $(10.7 \pm 2.3 \mathrm{k} \Omega$ at $1 \mathrm{kHz})$ was less than cloudy PEDOT $(33.1 \pm 4.1 \mathrm{k} \Omega$ at $1 \mathrm{kHz})$, the effective surface area was increased by a combination of PEDOT NTs on the surface of electrode sites as well as cloudy PEDOT inside the alginate (PEDOT NTs $+\mathrm{HG}+\mathrm{PEDOT}$ ), which produced the minimum impedance. Table 1 shows the impedance value at $1 \mathrm{kHz}$ for different coatings. The initial impedance of the bare gold sites was $783 \pm 15.7 \mathrm{k} \Omega$, which decreased to a minimum of $2.5 \pm 0.5 \mathrm{k} \Omega$ after PEDOT deposition around electrospun nanofibers and within the hydrogel matrix (PEDOT NTs + HG + PEDOT). This dramatic change is presumably due to substantially increase in the effective surface area. 

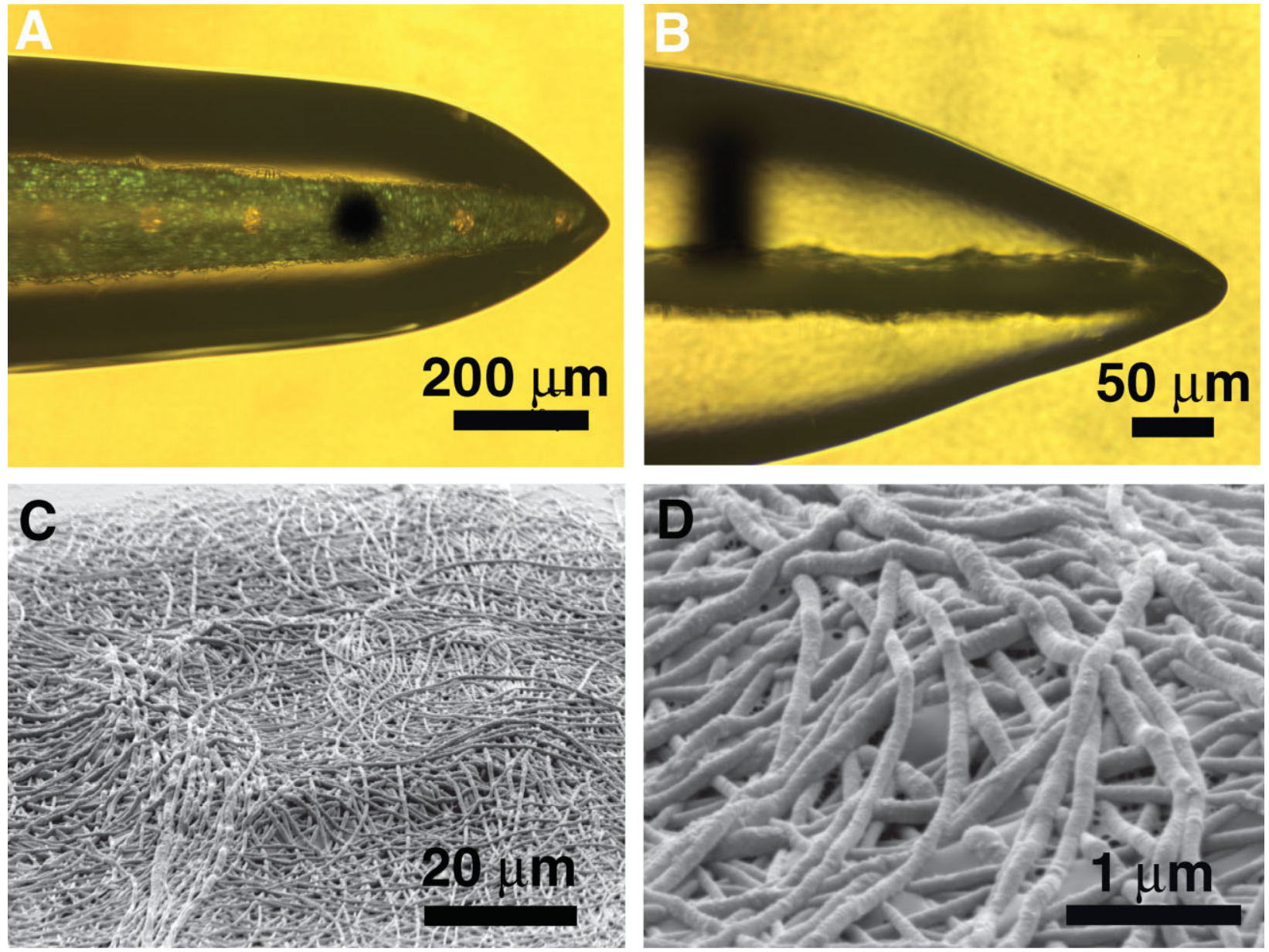

Figure 6. A) Optical micrograph of deposited PEDOT (black color) on electrode site (top view), B) side view of (A) showing vertical growth of PEDOT from an electrode site and through the alginate hydrogel scaffold (black color), C) SEM images of electrode site after dissolving the alginate coating and electrospun nanofibers. This image reveals that PEDOT was grown around the electrospun nanofibers to form PEDOT NTs. D) Higher magnification image of (C).

Ludwig et al. reported chronic neural recording using modified microelectrodes with PEDOT. ${ }^{[56]}$ Surfactant-templated PEDOT was deposited on $703-\mu \mathrm{m}^{2}$ electrode sites and reduced impedance of electrode sites from 900 to $60 \mathrm{k} \Omega$. Their results showed that the quality of signal recording in rats was improved. ${ }^{[56]}$ Since cloudy PEDOT in hydrogel in combination with nanotubes (PEDOT NTs + HG + PEDOT) has an even lower impedance, our EIS measurements suggest that even higher quality signals should be recorded if these materials were used in vivo. By testing the impedance of neighboring sites that were 200- $\mu \mathrm{m}$ apart, we found that the cloud-like PEDOT did not connect the electrode sites together.

\subsection{Cyclic Voltammetry (CV)}

CV was used to explore the charge capacity per unit area (charge capacity density or CCD) through the electrode sites after surface modification with PEDOT NTs, cloudy PEDOT inside hydrogel $(\mathrm{HG}+\mathrm{PEDOT})$, and PEDOT NTs in combination with cloudy PEDOT inside the hydrogel (PEDOT NTs + HG + PEDOT). The

electrode sites were swept from $-0.9-0.5 \mathrm{~V}$ at a scan rate of $100 \mathrm{mVs}^{-1}$ versus Ag/AgCl reference electrode. During oxidation or reduction of PEDOT, the polymer undergoes expansion or contraction, which is associated with migration of counter ions (doping) and cations either toward the solution or toward the PEDOT (depending on the size of the counter ions). ${ }^{[26]}$ The oxidation and reduction reactions involve current flow through PEDOT that appears as peaks (Ipc and Epc, corresponding to cathodic reduction current and potential, Ipa and Epa corresponding to anodic oxidation current and potential). Although PEDOT electropolymerization was done at the same applied charge density $2.88 \mathrm{C} \mathrm{cm}^{-2}$, the absolute value of current peaks (|Ipc| and |Ipa|) varies in the following order: PEDOT $\mathrm{NTs}+\mathrm{HG}+\mathrm{PEDOT}>$ PEDOT NTs $>$ HG + PEDOT (Fig. 7H). We also observed slight changes in oxidation and reduction potential for different coatings that have been recorded and described through literature for PEDOT derivatives. ${ }^{[37,38]}$ The surface area under the CV curve is proportional to the CCD that a particular coating material can transfer (store) during one cycle of $\mathrm{CV}$. The CCD is calculated by dividing the product of the voltage and the time (pulse width) by the product of the impedance and geometric surface area of the electrode. 


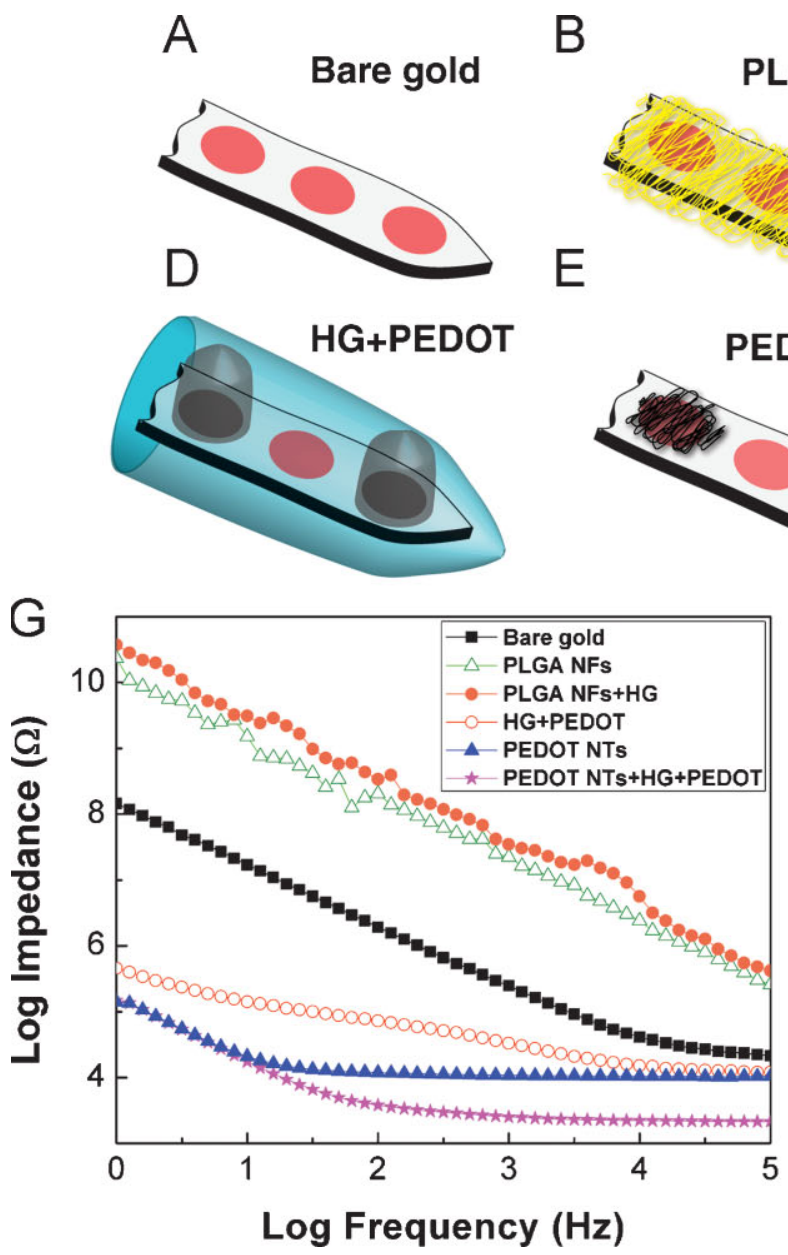

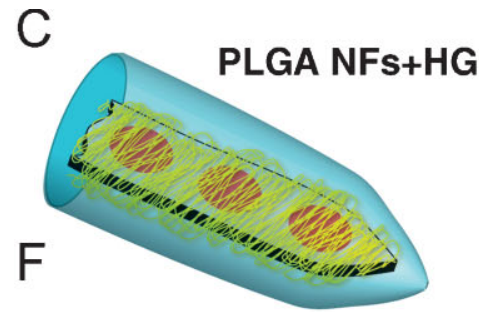

PLGA NFs

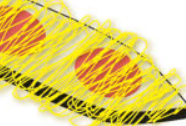

PEDOT NTS
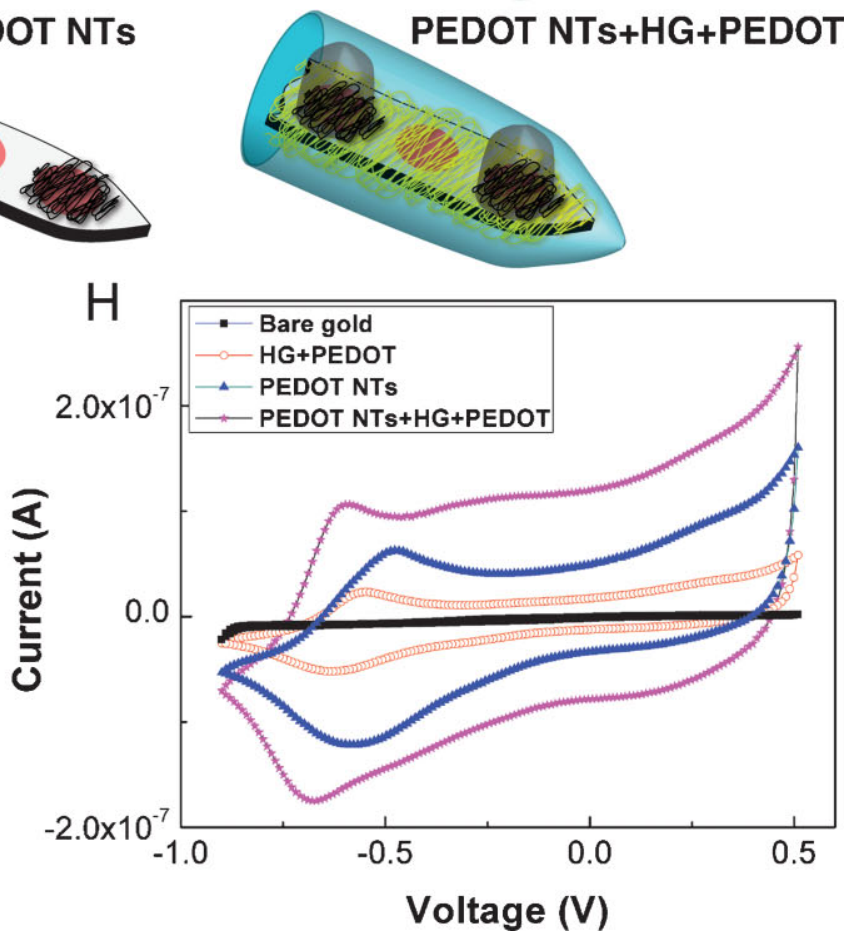

Figure 7. Schematic illustration of A) uncoated electrodes, B) DEX-loaded electrospun PLDL75C25A nanofibers on the electrodes (PLGA NFs), C) alginate hydrogel coating of DEX-loaded electrospun PLDL75G25A nanofibers on the electrodes (HG + PLGA NFs), D) cloudy PEDOT inside the alginate hydrogel on particular electrodes (HG + PEDOT), E) PEDOT NTs on the electrodes (PEDOT NTs), F) PEDOT NTs and cloudy PEDOT inside the hydrogel on the electrodes (PEDOT NTs + HG + PEDOT), G) EIS of bare gold (black squares), PLGA NFs (green hollow triangles), PLGA NFs + HG (orange circles), HG + PEDOT (red hollow circles), PEDOT NTs (blue triangles), and PEDOT NTs + HG + PEDOT (pink stars), with the applied deposition charge density was $2.88 \mathrm{C} \mathrm{cm}^{-2}, \mathrm{H}$ ) CV of bare gold (black squares), HG + PEDOT (red hollow circles), PEDOT NTs (blue triangles), and PEDOT NTs + HG + PEDOT (purple stars); the potential was swept from -0.9 to $0.5 \mathrm{~V}$ at a scan rate of $100 \mathrm{mV} \mathrm{s}^{-1}$.

Table 1. Electrical properties of different coating materials: bare gold, DEX-loaded electrospun PLDL75G25A (PLGA) nanofibers on the electrodes (PLGA NFs), alginate hydrogel coating of DEX-loaded electrospun PLGA NFs on the electrodes (HG + PLGA NFs), cloudy PEDOT inside the alginate hydrogel on the electrodes (HG + PEDOT), PEDOT NTs on the electrodes (PEDOT NTs), PEDOT NTs and cloudy PEDOT inside the hydrogel on the electrodes (PEDOT NTs + HG + PEDOT) [a]

\begin{tabular}{|c|c|c|c|c|c|c|}
\hline & Impedance $[k \Omega]$ & $\mathrm{CCD}\left[\mathrm{mC} \mathrm{cm}{ }^{-2}\right]$ & Epc [V] & Epa [V] & $\mathrm{Ipc}[\mathrm{nA}]$ & Ipa $[\mathrm{nA}]$ \\
\hline Bare gold & $783.3 \pm 15$ & $1.28 \pm 0.6$ & $N A[b]$ & NA & NA & NA \\
\hline PLLA NFs & $2246.7 \pm 23$ & NA & NA & NA & NA & NA \\
\hline $\mathrm{HG}+$ PLLA NFs & $3472.4 \pm 19$ & NA & NA & NA & NA & NA \\
\hline HG + PEDOT & $33.1 \pm 4$ & $41.2 \pm 2.8$ & -0.56 & -0.38 & 23.1 & -52.1 \\
\hline PEDOT NTs & $10.7 \pm 2$ & $112.4 \pm 9.1$ & -0.48 & -0.59 & 62.4 & 126.7 \\
\hline PEDOT NTs + HG + PEDOT & $2.5 \pm 0.5$ & $223.8 \pm 6.4$ & -0.60 & -0.68 & 113.2 & 175.4 \\
\hline
\end{tabular}

[a] PEDOT was electrochemically polymerized on the neural microelectrodes with an applied charge density of $2.88 \mathrm{C} \mathrm{cm}{ }^{-2}$. Data are shown for $\pm \mathrm{standard}$ deviation ( $n=48)$. [b] NA = not available due to nonconductive coating layer. 
A threshold charge density (minimum) is required to generate neural excitation however there is a limit for charge density (maximum of $10 \mu \mathrm{C} \mathrm{cm}{ }^{-2}$ ), which causes damage to neural tissue. ${ }^{[57,58]}$ According to Table 1, the CCD during one cycle of CV (swept voltage between -0.9 and 0.5 ) for a $1250-\mu \mathrm{m}^{2}$ gold electrode was $1.28 \pm 0.6 \mathrm{mC} \mathrm{cm}^{-2}$. However, this value increased to $41.2 \pm 2.8 \mathrm{mC} \mathrm{cm}^{-2}$ for cloudy PEDOT inside hydrogel (HG + PEDOT), $112.4 \pm 9.1 \mathrm{mC} \mathrm{cm}{ }^{-2}$ for PEDOT NTs, and $223.8 \pm 6.4 \mathrm{mC} \mathrm{cm}{ }^{-2}$ for a combination of PEDOT NTs and cloudy PEDOT inside the hydrogel (PEDOT NTs + HG + PEDOT).

The electrodes of interest in this study were small diameter microelectrodes $(40 \mu \mathrm{m})$ designed primarily for local neural recording. However, given these dramatic changes in CCD found with our materials, we expect that they will also prove to be particularly important for large electrode stimulating devices such as deep brain stimulators, pacemakers, and peripheral nerve cuff electrodes.

\subsection{Weight Loss and Water Absorption of PLDL75G25A}

The mechanism of degradation in poly ( $\alpha$-hydroxy acids) such as PLLA and PLDL75G25A films and microstructures in aqueous media has been widely investigated. ${ }^{[59-62]}$ However, the degradation behavior of their nanofibers prepared by electrospinning has been less studied. ${ }^{[63,64]}$ Figure 8D shows the mass loss and water uptake of electrospun PLDL75G25A nanofiber mat. The in vitro degradation mechanism of water insoluble bioresorbable poly ( $\alpha$-hydroxy acids) is hydrolytic degradation. This degradation proceeds more rapidly in the center than at the surface as the
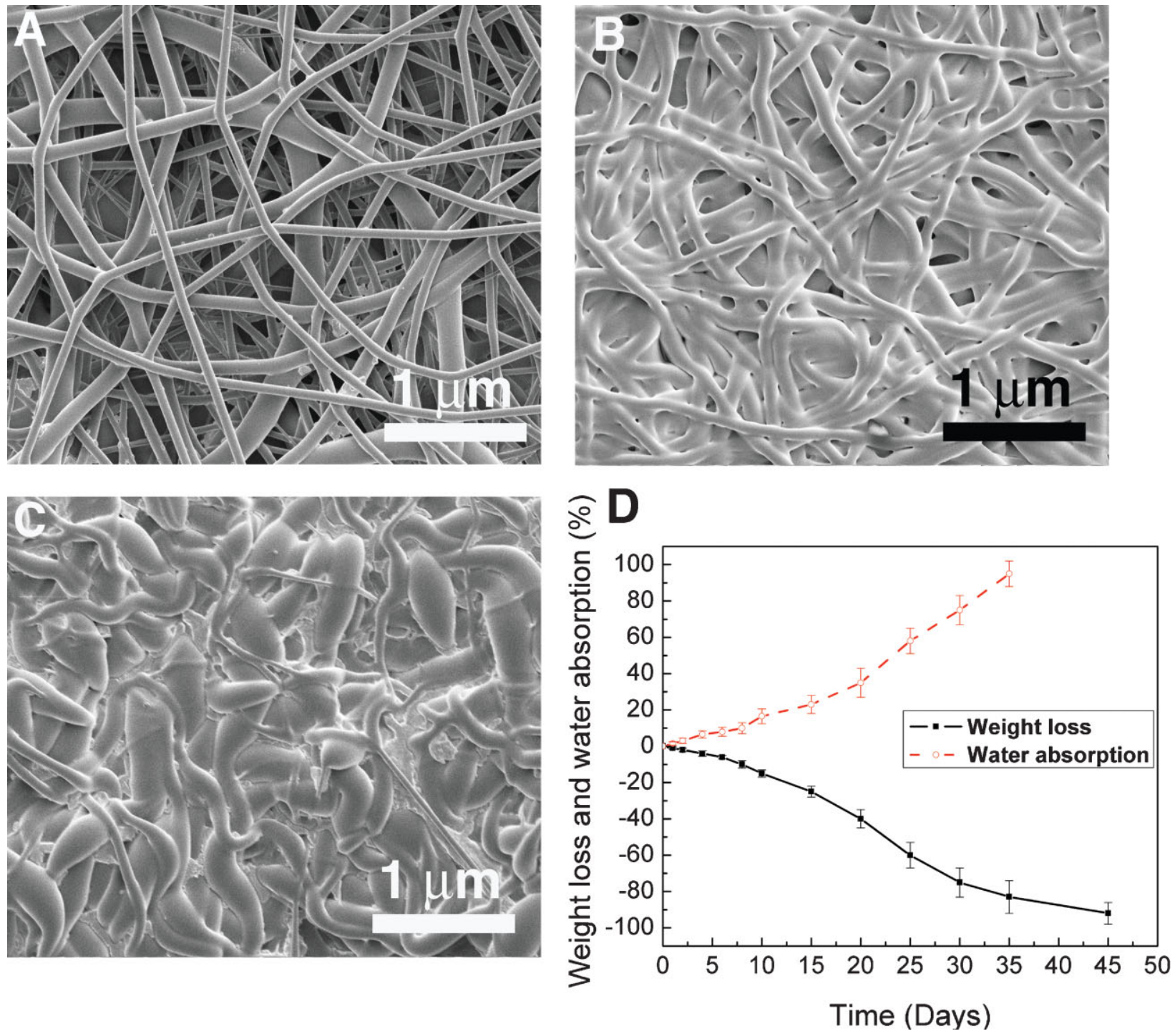

Figure 8. SEM images of electrospun PLDL75G25A nanofibers showing the degradation of nanofibers in PBS solution $\left(\mathrm{pH}=7.4, T=37^{\circ} \mathrm{C}\right)$. A) After 6 days, no significant morphological changes were observed. B) After 15 days, the swollen fibers started diffusing and creating massive bulk of polymer. C) After 5 weeks, only diffused fibers and of some piece of degraded materials were left. D) Percent weight loss and water absorption of PLDL75C25A nanofibers during in vitro degradation. Data are shown for \pm standard deviation $(n=10)$. 
water penetrate into the polymer. ${ }^{[65]}$ In contrast to semicrystalline PLLA, PLDL75G25A is an amorphous polymer allowing greater absorption of water into the polymer and faster degradation. ${ }^{[6]}$ During the first 6 days of incubation (this time period was defined as the induction period), PLDL75G25A exhibited a very slow weight loss rate. It was reported that once poly $(\alpha$-hydroxy acids) are placed in an aqueous medium, they absorb water and hydrolytic cleavage of the ester bonds starts with decreasing molecular weight. ${ }^{[61]}$ At this early stage, degradation is occurring predominantly at the surface of the nanofibers rather than in the bulk, because of the gradient in concentration of the absorbed water. ${ }^{[67]}$ The mass loss was accelerated after this period because of even more ester bonds cleavage due to absorption of water. About $30 \%$ of weight loss was observed within 2 weeks of degradation (Fig. 8D). Since the swollen nanofibers began diffusing together and creating a massive polymer (Fig. 8B and C), the weight loss was decelerated after 2 weeks (Fig. 8D). Due to the unique nanofiber morphology with extremely high surface area to volume ratio, the electrospun PLDL75G25A nanofiber mat absorbed about $20 \mathrm{wt} \%$ of water after 10 days of incubation. In contrast, it took more than 2 weeks for poly (glycolic acid) (PGA) solid films to achieve the same water absorption level. ${ }^{[68]}$

However, the water content increased dramatically after 15 days of degradation. For example, the water uptake reached a $100 \mathrm{wt} \%$ level of the initial sample weight after 35 days. It was too difficult to measure the water absorption after day 35 because of too much chain cleavage and loss of molecular weight of the polymer. The corresponding mass loss after 35 days was more than $90 \mathrm{wt} \%$, which was faster than that of the cast solid film. ${ }^{[69]}$ The water uptake could be attributed both to the formation of a porous surface in the nonwoven membrane and to the swelling of degraded products. Figure $8 \mathrm{~B}$ and $\mathrm{C}$ illustrates the morphological changes of the electrospun PLDL75G25A nanofiber membrane during in vitro degradation. No significant morphological changes were observed in the first 6 days of incubation (Fig. 8A). However, the swollen fibers started diffusing and creating massive bulk of polymer after 15 days of degradation (Fig. 8B). After 5 weeks of degradation, only diffused fibers and of some piece of degraded materials were left (Fig. 8C).

\subsection{Dexamethasone Release Profile}

In this study, DEX as an anti-inflammatory drug was incorporated in electrospun nanofibers. DEX, a synthetic corticosteroid is widely used in treatment of neurological disease, especially to reduce cerebral edema associated with tumors. ${ }^{[70]}$ Because of its limited ability to cross the blood-brain barrier, DEX needs to be administered in high systemic dosages to achieve therapeutic levels in the brain. However, systemic administration of DEX is associated with serious side effects. ${ }^{[42,43]}$ To accomplish this, we first incorporated DEX within biodegradable nanofibers and finally coated them with alginate hydrogel. Poly(esters) based on poly(lactic acid), poly(glycolic acid), and their copolymers PLGA have been widely used as biocompatible polymeric materials for controlled drug delivery systems by the incorporation of drugs into their polymer matrices. ${ }^{[45,49,51,71]}$ This polymer protection can involve delaying the dissolution of drug molecules and controlling the rate of drug release via hydrolytic degradation of the polymer matrix. Figure 9 shows DEX release profiles from PLLA, PLDLA, PLDL75G25A, and alginate-coated PLDL75G25A nanofibers. PLLA is a high molecular weight semi-crystalline polymer and formation of PLLA spun fibers increases the crystallinity of PLLA. ${ }^{[72]}$ In contrast, PLDLA and PLDL75G25A are lower molecular weight and amorphous polymers. Since, increasing the molecular weight and crystallinity causes a decrease in the degradation rate, PLLA degrades significantly slower than PLDLA and PLDL75G25A. ${ }^{[60-62,65,73,74]}$ There was a burst of about $6 \%$ during first week of incubation of PLLA in PBS and only about $10 \%$ of DEX was released after 41 days (Fig. 9A). Since PLDLA is more hydrophobic than PLDL75G25A, the DEX release profile from PLDL75G25A is faster than PLDLA (Fig. 9B). As illustrated in Figure 10A-C, a significant initial release of accumulated drug on the surface of nanofibers (burst effect) due to an initial surface erosion followed by a slow release of DEX was observed for all polymers; this phenomenon has been frequently reported. ${ }^{[75,76]}$ The burst effect may be primarily caused by an imperfect entrapment of the drug in the polymer matrix or their tendency to migrate to the nanofiber surface during the electrospinning process (Fig. 10A). As the nanofibers are placed in an aqueous environment, at the initial stage water penetrates into the surface and a large amount of DEX is released due to the initial surface erosion (Fig. 10B). Subsequently, water penetrates into the bulk of the nanofibers causing polymer bond cleavage and bulk erosion, generating interconnecting pores and channels within nanofibers that allow the remaining DEX to be released (Fig. 10C).

As illustrated in Figure 1C, the DEX-loaded biodegradable nanofibers of PLDL75G25A were covered by alginate hydrogel on the surface of the neural electrode. Controlled release of proteins and drugs from alginate hydrogel matrices has been reported. ${ }^{[77-79]}$ The release mechanism of drugs from hydrogels such as alginate is diffusion controlled. This means the drugs must diffuse within an aqueous solution through the tortuous pathways of the hydrogel network in order to exit from the matrix. Figure 9B and $C$ show sustained release of DEX from PLDL75G25A after coating with alginate hydrogel during $1000 \mathrm{~h}$ and $20 \mathrm{~h}$ of incubation in PBS solution, respectively. Since the samples were dehydrated before placing in PBS solution, the initial release of DEX was delayed about $4 \mathrm{~h}$ due to swelling and water uptake of alginate (Fig. 9C). Also, the release burst effect and release profile of DEX was reduced by about $40 \%$. As shown schematically in Figure 10D and E, the water insoluble DEX molecules that are released from nanofibers need to diffuse through the hydrophilic alginate matrix. Therefore, the trapped DEX in alginate releases into the media slower than from the nanofibers alone. In case of PLDL75G25A nanofibers, approximately $95 \%$ of DEX was released in 5 weeks whereas for the entrapped PLDL75G25A nanofibers in alginate matrix, only $60 \%$ of DEX was released after 5 weeks (Fig. 9B). The advantage of composite hydrogel-biodegradable polymer coatings is that there can be a more prolonged and sustained release of drug due to the two mechanisms of release: delayed dissolution of the nanofibers and diffusion through the hydrogel. This makes it possible to control the delivery of the DEX without a burst effect. At the beginning, the drug will be released into the hydrogel matrix due to degradation of PLDL75G25A nanofibers, followed by slower release of drug as it diffuses through the alginate hydrogel matrix. 


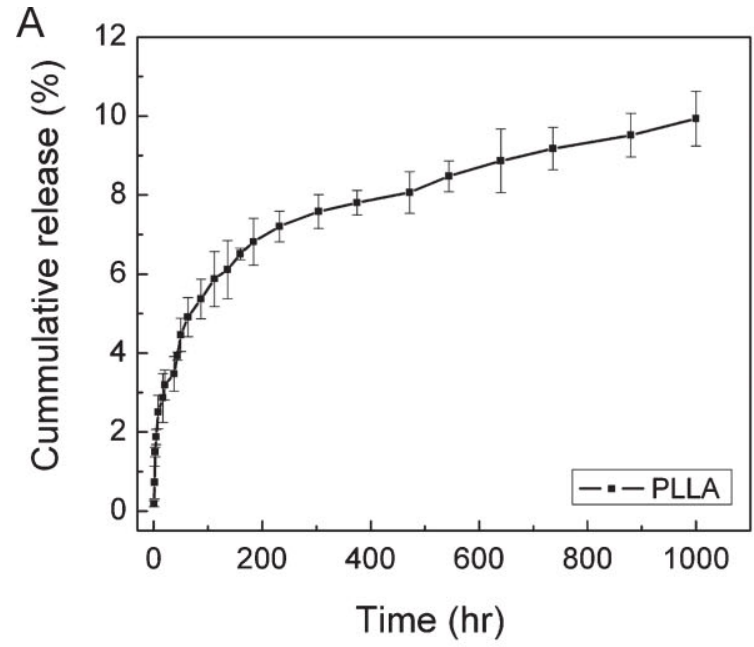

B
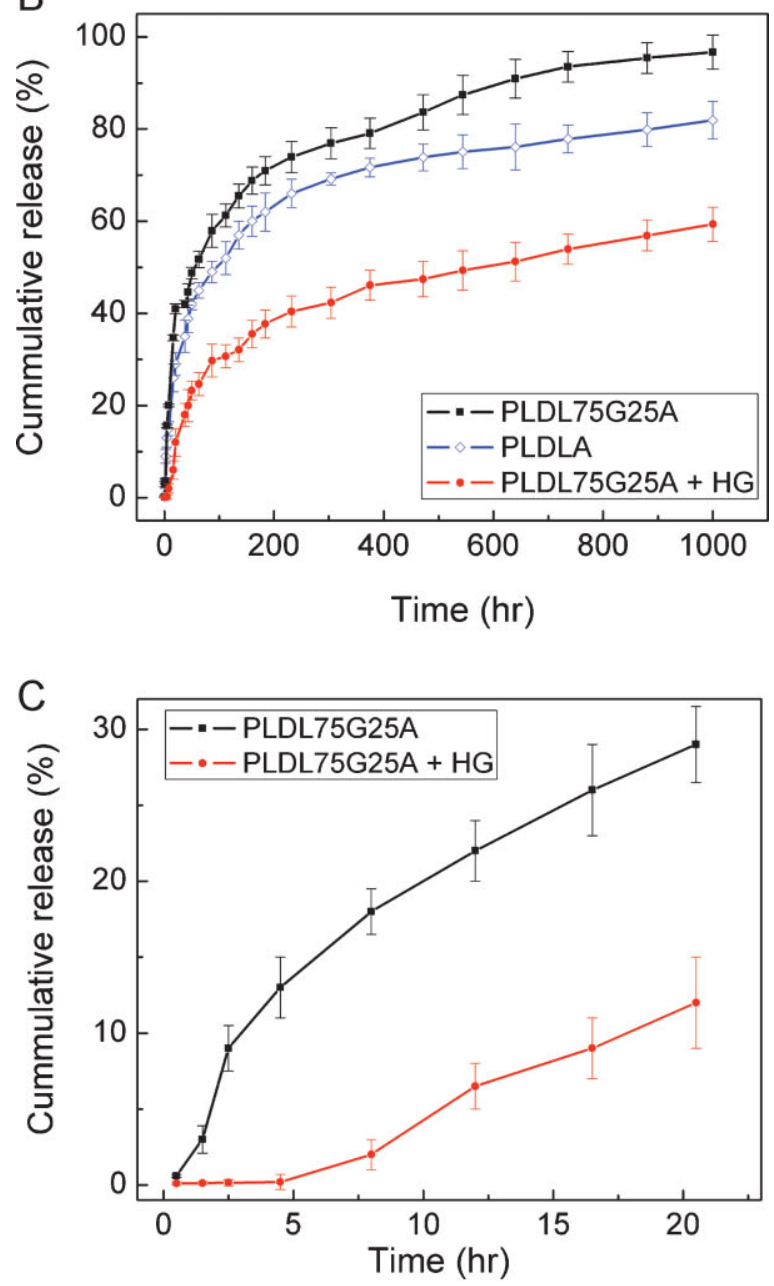

Figure 9. Percentage cumulative mass release profiles of DEX-loaded A) PLLA nanofibers (black squares) over a time span of $1000 \mathrm{~h}, \mathrm{~B})$ PLDLA nanofibers (blue hollow circles), PLDL74G25A nanofibers (black squares), and alginate hydrogel-coated PLDL74G25A nanofibers (red circles), over $1000 \mathrm{~h}, \mathrm{C}$ ) a zoom window of (B) for PLDL74G25A nanofibers (black squares), and alginate hydrogel-coated PLDL74C25A nanofibers (red circles), over $20 \mathrm{~h}$. Data are shown for \pm standard deviation $(n=20)$.

\section{Conclusions}

We have established methods for the fabrication of multifunctional nanobiomaterials that can be used for coating neural microelectrode arrays. The process includes electrospinning of DEX-incorporated biodegradable nanofibers, encapsulation of these nanofibers by an alginate hydrogel layer, and then electrochemical polymerization of the conducting polymer PEDOT on the electrode site, around the DEX-loaded nanofibers, and within the hydrogel matrix. We have shown that electrical properties of neural microelectrodes have been significantly improved with these coatings. The impedance of the electrode sites significantly decreased, especially at $1 \mathrm{kHz}$ from $783 \pm 15.7$ to $2.5 \pm 0.5 \mathrm{k} \Omega$ (about two orders of magnitude). The CCD

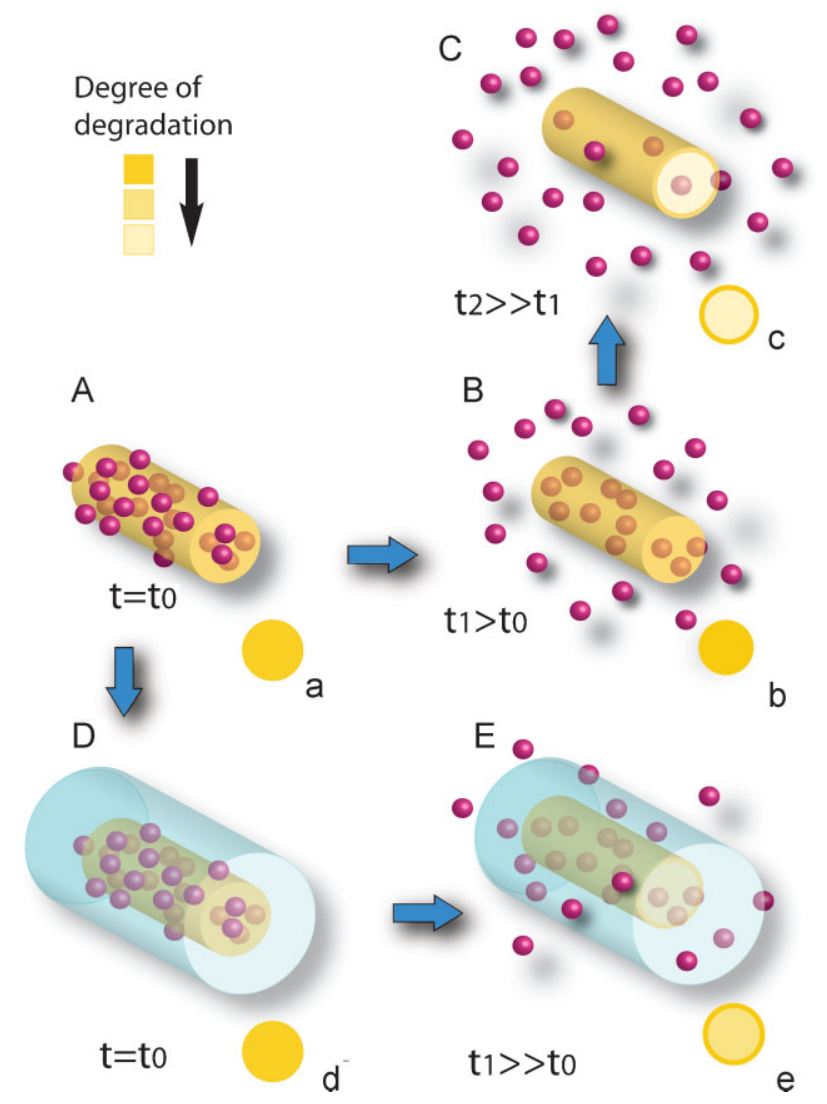

Figure 10. Schematic illustration showing proposed mechanism of DEX release and degree of degradation of electrospun nanofibers. A) DEXloaded electrospun nanofibers showing accumulation of drug molecules at the surface of nanofibers before placing in aqueous solution $\left(t=t_{0}\right)$, a) cross-section of nanofibers with surface area $A_{a}, B$ ) fast release of DEX from surface of nanofibers (burst effect) after placing in aqueous solution $\left(t_{1}>t_{0}\right)$ due to initial surface erosion of nanofibers, b) crosssection of nanofibers with surface area $A_{b}\left(A_{a}>A_{b}\right)$. C) Slow release of drug due to bulk erosion or delayed dissolution mechanism at $t_{2} \gg t_{1}, \mathrm{c}$ ) crosssection of nanofibers with surface area $A_{c}, D$ ) alginate hydrogel coating of DEX-loaded electrospun nanofibers shown in (A) before placing in aqueous solution $\left(t=t_{0}\right)$, d) cross-section of nanofibers with surface area $A_{d}$ $\left.\left(A_{d}=A_{a}\right), E\right)$ sustained release of drug from alginate hydrogel using delayed dissolution and diffusion mechanism at $t_{1} \gg t_{0}$, e) cross-section of nanofibers with surface area $A_{e}\left(A_{e}<A_{c}\right)$ due to slower degradation of nanofibers. 
significantly increased from $1.28 \pm 0.6$ to $223.8 \pm 6.4 \mathrm{mC} \mathrm{cm}^{-2}$. The surface morphology and degradation rate of PLDL75G25A has been studied and the release profile of DEX from PLLA, PLDLA, and PLDL75G25A has been investigated. It has been demonstrated that alginate hydrogel coatings could not only decrease the burst effect of DEX release for controlling the longterm release patterns but also create a matrix for growing PEDOT within the alginate in order to increase the conductivity of electrode sites. This method provides a generally useful means for creating soft, low impedance, high charge density, controlled releasing for neural prostheses, and other biosensor applications.

\section{Experimental}

Materials: High molecular weight PLLA (RESOMER L 210) with inherent viscosity 3.3-4.3 $\mathrm{dl} \mathrm{g}^{-1}$ was purchased from Boehringer Ingelheim Pharma GmbH and Co. (KG, Germany). PLDL75G25A (Medisorb 7525 DL, low I.V., inherent viscosity $0.6-0.7 \mathrm{dl} \mathrm{g}^{-1}, \mathrm{MW} 92 \mathrm{kDa}$ ) and PLDLA (Medisorb $100 \mathrm{DL}$, low I.V., inherent viscosity $0.7-0.8 \mathrm{dl} \mathrm{g}^{-1}$, MW $100 \mathrm{kDa}$ ) was obtained from Alkermes Inc. (Cincinnati, Ohio). DEX (MW $392.5 \mathrm{~g}$ $\mathrm{mol}^{-1}$ ) was purchased from Alexis Corporation. 3,4-ethylenedioxythiophene (EDOT, BAYTRON M) with molecular weight $142.17 \mathrm{~g} \mathrm{~mol}^{-1}$ was received from $\mathrm{H}$. C. Starck Inc. (Newton, MA). Lithium perchlorate $\left(\mathrm{LiClO}_{4}\right)$ was purchased and used as received from Aldrich. Sodium alginate (MVG) was purchased from Pronova Biomedical (Norway) with an overall guluronic acid (G-block) content of approximately $60 \%$, as reported by the manufacturer. Sterile phosphate buffered saline (PBS) was purchased from Hyclone (cell culture media, $0.1 \mu \mathrm{m}$ sterile filtered).

Fabrication of Electrospun Nanofiber Templates: PLLA, PLDLA, and PLDL75G25A nanofibers loaded with DEX were prepared by electrospinning of polymer/drug solutions. PLLA/DEX solution was prepared by dissolving a mixture of PLLA $(0.4 \mathrm{~g})$ and DEX $(0.1 \mathrm{~g})$ in chloroform $(6.4 \mathrm{~mL})$ at a temperature of $50^{\circ} \mathrm{C}$ for $10 \mathrm{~h}$ in order to have a homogenous solution with PLLA concentration of $3.96 \%$ (w/w), DEX concentration of $0.99 \%$, and ratio of PLLA/DEX:4:1. In case of PLDL75C25A and PLDLA, a mixture of polymer $(1.7 \mathrm{~g})$ and DEX $(0.17 \mathrm{~g})$ was added to chloroform $(6.4 \mathrm{~mL})$ then the solution was stirred at a temperature of $55^{\circ} \mathrm{C}$ for $10 \mathrm{~h}$ to prepare a homogeneous solution with concentration of $14.82 \% \mathrm{w} / \mathrm{w}$ polymer and $1.48 \% \mathrm{w} / \mathrm{w}$ DEX and ratio of polymer/DEX:10:1. DEX-loaded nanofibers were directly deposited on the microfabricated electrode arrays by electrospinning (Fig. 2). The electrospinning process was carried out in an electrical field of $0.6 \mathrm{kV} \mathrm{cm}^{-1}$ with flow rate of $0.25 \mathrm{~mL} \mathrm{~h}^{-1}$ for $1 \mathrm{~min}$. The neural probes were held at a distance of $11 \mathrm{~cm}$ from the syringe needle.

Preparation of Alginate Coating: An aqueous alginate solution was prepared by dissolving MVG in deionized water. The alginate concentration in the solution was $1 \% \mathrm{w} / \mathrm{w}$. Hydrogel coating was then formed around the electrode shank by dipping the shank sequentially in both alginate solution and $\mathrm{CaCl}_{2}(0.1 \mathrm{M})$ solution. The hydrogel was dried by exchanging the water in the gel by ethanol followed by drying in an air stream.

Electrochemical Deposition of Conducting Polymers: The electrochemical deposition was performed on an individual electrode site of the eightchannel acute neural electrode with $1250-\mu \mathrm{m}^{2}$ gold recording sites by an Autolab PGSTAT-12 (EcoChemie, Utrecht, Netherlands) in galvanostatic mode with a conventional two-electrode configuration at room temperature. Conducting polymer deposition was carried out in EDOT $(0.01 \mathrm{M})$ and $\mathrm{M} \mathrm{LiClO}_{4}(0.1 \mathrm{M})$ aqueous solution at a current density of $0.5 \mathrm{~mA} \mathrm{~cm}{ }^{-2}$ for $30 \mathrm{~min}$. The amount of polymer coated on the electrode site was controlled by the total charge passed during polymerization. The working electrode was connected to the electrode site. The reference and counter electrodes were connected to a platinum wire within the EDOT/LiClO ${ }_{4}$ solution. PEDOT was polymerized on total 144 electrode sites for each of the following samples: i) alginate hydrogel-coated electrode (HG + PEDOT) (Fig. 7D), ii) electrospun nanofibers (PEDOT NTs) (Fig. 7E), iii) electrospun nanofibers and alginate hydrogel-coated electrode (PEDOT NTs + HG + PEDOT) (Fig. 7F).

Dissolving Alginate Hydrogel and Electrospun Nanofibers for SEM: To dissolve the alginate hydrogel layer, neural electrodes were immersed into deionized water for $24 \mathrm{~h}$. Afterward they were placed into chloroform for $1 \mathrm{~min}$ to remove the electrospun nanofibers.

Electrochemical Impedance Spectroscopy: An Autolab PGSTAT-12 and Frequency Response Analyzer (FRA) software were used to record impedance spectra of electrode sites. A solution of $0.1 \mathrm{M} \mathrm{PBS}(\mathrm{pH}=7)$ was used as an electrolyte in a three-electrode cell. The working electrode was connected to electrode site through a connector. The counter electrode was connected to a platinum foil that was placed in a glass container. An $\mathrm{Ag} / \mathrm{AgCl}$ reference electrode and the neural microelectrode tip were immersed in glass container of electrolyte. An AC sinusoidal signal of $5 \mathrm{mV}$ in amplitude was used to record the impedance over a frequency range of $1-10^{5} \mathrm{~Hz}$.

Cyclic Voltammetry (CV): CV was performed using an Autolab PGSTAT12 instrument in a three-electrode configuration as described earlier. A scan rate of $100 \mathrm{mV} \mathrm{s}^{-1}$ was used and the potential on the working electrode was swept between -0.9 and $0.5 \mathrm{~V}$. All the potentials are reported versus the $\mathrm{Ag} / \mathrm{AgCl}$ reference electrode. Before each CV curve was recorded, several cycles were swept to insure that the PEDOT had reached a stable state. The GPES software (EcoChemie, Utrecht, Netherlands) was used to estimate the total CCD during one cycle of CV.

Degradation Studies: Electrospun fiber mats were cut into a total of ten rectangular shapes with dimensions of $20 \times 20 \times 30 \mathrm{~mm}^{3}$ for in vitro degradation studies. The electrospun specimens were dipped into $70 \%$ ethanol for $20 \mathrm{~s}$ to minimize the potential for bacterial contamination and placed into sterile, closed bottles containing sterile PBS $(100 \mathrm{~mL})(\mathrm{pH}$ 7.27), and incubated in vitro at a temperature of $37.0 \pm 0.7^{\circ} \mathrm{C}$ for different periods of time. For the in vitro degradation study, four specimens were recovered at the end of each degradation period and dabbed dry with a tissue, and each sample was weighed immediately $\left(W_{w}\right)$. The samples were then dried in a vacuum oven at room temperatures for 1 week and were weighed again $\left(W_{d}\right)$. The mass loss and the water content percentages of the samples were calculated with the following equations, based on the initial mass of each sample $\left(W_{0}\right)$ before incubation:

$\operatorname{mass} \operatorname{loss} \%=\frac{\left(\mathrm{W}_{\mathrm{d}}-\mathrm{W}_{0}\right)}{\mathrm{W}_{0}} \times 100$

water content $\%=\frac{\left(\mathrm{W}_{\mathrm{w}}-\mathrm{W}_{\mathrm{d}}\right)}{\mathrm{W}_{0}} \times 100$

In Vitro Drug Release Study: The release of DEX from PLLA, PLDLA, and PLDL75C25A nanofibers was monitored as a function of incubation time in PBS at $37^{\circ} \mathrm{C}$. The samples were immersed in PBS solution $(\mathrm{pH}=7.4)$. At specific times the concentration of DEX was determined in the solution by a DU UV/Vis Spectrophotometer (Beckman Coulter Inc.) at $242 \mathrm{~nm}$.

\section{Acknowledgements}

This work was supported by NIH-NINDS-NO1-NS-1-2338. The authors would like to acknowledge the University of Michigan Center for Neural Communication Technology (CNCT), Army Research Office MURI (Contract no. W911NF-06-1-0218, Proposal no. 50376-LS-MUR). Eugene Dariush Daneshvar and Kip Ludwig provided helpful comments on the manuscript. D. C. M. is a founder and chief scientific officer of Biotectix, a University of Michigan spin-off company that is actively interested in the commercialization of soft, low impedance, bioactive coatings for biomedical devices. M. R. A. and D. C. M. are both co-inventors on 
invention disclosures and patent applications related to these materials that have been filed with the University of Michigan Office of Technology Transfer and the US Patent and Trademark Office.

Received: October 10, 2008

Revised: November 13, 2008 Published online: January 29, 2009

[1] J. C. Williams, R. L. Rennaker, D. R. Kipke, Brain Res. Protoc. 1999, 4, 303.

[2] K. L. Drake, K. D. Wise, J. Farraye, D. J. Anderson, S. L. Bement, IEEE Trans. Biomed. Eng. 1988, 35, 719.

[3] D. R. Kipke, R. J. Vetter, J. C. Williams, J. F. Hetke, IEEE Trans. Neural Syst. Rehab. Eng. 2003, 11, 151.

[4] P. J. Rousche, R. A. Normann, J. Neurosci. Methods 1998, 82, 1.

[5] K. D. Wise, D. J. Anderson, J. F. Hetke, D. R. Kipke, K. Najafi, Proc. IEEE 2004, 92, 76.

[6] G. T. A. Kovacs, in Enabling Technologies for Cultured Neural Networks, (Eds: D.A. Stenger, T. M. McKenna), Academic Press, London, UK 1994, p. 121.

[7] D. A. Robinson, Proc. Inst. Electr. Electron. 1968, 56, 1065.

[8] D. J. Edell, V. V. Toi, V. M. McNeil, L. D. Clark, IEEE Trans. Biomed. Eng. 1992, 39, 635

[9] V. S. Polikov, P. A. Tresco, W. M. Reichert, J. Neurosci. Methods 2005, 148, 1.

[10] D. H. Szarowski, M. D. Andersen, S. Retterer, A. J. Spence, M. Isaacson, H. G. Craighead, J. N. Turner, W. Shain, Brain Res. 2003, 983, 23.

[11] J. N. Turner, W. Shain, D. H. Szarowski, M. Andersen, S. Martins, M. Isaacson, H. Craighead, Exp. Neurol. 1999, 156, 33.

[12] M. A. L. Nicolelis, D. Dimitrov, J. M. Carmena, R. Crist, G. Lehew, J. D. Kralik, S. P. Wise, Proc. Natl. Acad. Sci. USA 2003, 100, 11041.

[13] P. J. Rousche, D. S. Pellinen, D. P. Pivin, J. C. Williams, R. J. Vetter, D. R. Kipke, IEEE Trans. Biomed. Eng. 2001, 48, 361.

[14] K. K. Lee, J. P. He, A. Singh, S. Massia, G. Ehteshami, B. Kim, G. Raupp, J. Micromech. Microeng. 2004, 14, 32.

[15] A. Singh, G. Ehteshami, S. Massia, J. P. He, R. G. Storer, G. Raupp, Biomaterials 2003, 24, 5083.

[16] J. Csicsvari, D. A. Henze, B. Jamieson, K. D. Harris, A. Sirota, P. Bartho, K. D. Wise, G. Buzsaki, J. Neurophysiol. 2003, 90, 1314.

[17] E. M. Maynard, E. Fernandez, R. A. Normann, J. Neurosci. Methods 2000, $97,93$.

[18] X. Y. Cui, D. C. Martin, Sens. Actuators, A 2003, 103, 384.

[19] S. F. Cogan, T. D. Plante, J. Ehrlich, "Sputtered iridium oxide films (SIROFs) for low-impedance neural stimulation and recording electrodes", presented at 26th Annual International Conference of the IEEE Engineering in Medicine and Biology Society, San Francisco, CA 2004.

[20] K. J. Otto, M. D. Johnson, D. R. Kipke, IEEE Trans. Biomed. Eng. 2006, 53, 333.

[21] X. Y. Cui, J. Wiler, M. Dzaman, R. A. Altschuler, D. C. Martin, Biomaterials 2003, 24, 777.

[22] L. Kam, W. Shain, J. N. Turner, R. Bizios, Biomaterials 2002, 23, 511.

[23] W. Shain, L. Spataro, J. Dilgen, K. Haverstick, S. Retterer, M. Isaacson, M. Saltzman, J. N. Turner, IEEE Trans. Neural Syst. Rehab. Eng. 2003, 11, 186.

[24] L. Spataro, J. Dilgen, S. Retterer, A. J. Spence, M. Isaacson, J. N. Turner, W. Shain, Exp. Neurol. 2005, 194, 289.

[25] N. K. Guimard, N. Gomez, C. E. Schmidt, Prog. Polym. Sci. 2007, 32, 876.

[26] E. Smela, Adv. Mater. 2003, 15, 481.

[27] J. Isaksson, P. Kjall, D. Nilsson, N. D. Robinson, M. Berggren, A. RichterDahlfors, Nat. Mater. 2007, 6, 673.

[28] R. A. Green, N. H. Lovell, G. G. Wallace, L. A. Poole-Warren, Biomaterials 2008, 29, 3393.

[29] G. Wallace, G. Spinks, Soft Matter 2007, 3, 665.

[30] M. R. Abidian, D. C. Martin, Biomaterials 2008, 29, 1273.

[31] M. R. Abidian, D. H. Kim, D. C. Martin, Adv. Mater. 2006, 18, 405.
[32] M. Berggren, A. Richter-Dahlfors, Adv. Mater. 2007, 19, 3201.

[33] X. Y. Cui, J. F. Hetke, J. A. Wiler, D. J. Anderson, D. C. Martin, Sens. Actuators, A 2001, 93, 8.

[34] J. Y. Yang, D. C. Martin, Sens. Actuators, B 2004, 101, 133.

[35] J. Y. Yang, K. Lipkin, D. C. Martin, J. Biomater. Sci. Polym. Ed. 2007, 18, 1075.

[36] M. Dietrich, J. Heinze, G. Heywang, F. Jonas, J. Electroanal. Chem. 1994, $369,87$.

[37] B. L. Groenendaal, F. Jonas, D. Freitag, H. Pielartzik, J. R. Reynolds, Adv. Mater. 2000, 12, 481

[38] L. Groenendaal, G. Zotti, P. H. Aubert, S. M. Waybright, J. R. Reynolds, Adv. Mater. 2003, 15, 855

[39] G. Heywang, F. Jonas, Adv. Mater. 1992, 4, 116.

[40] F. Jonas, L. Schrader, Synth. Met. 1991, 41, 831 .

[41] I. Winter, C. Reese, J. Hormes, G. Heywang, F. Jonas, Chem. Phys. 1995, 194, 207.

[42] B. J. Lipworth, Arch. Intern. Med. 1999, 159, 941.

[43] J. C. Melby, Ann. Intern. Med. 1974, 81, 505.

[44] R. A. Jain, Biomaterials 2000, 21, 2475.

[45] R. Langer, Science 1990, 249, 1527.

[46] J. Panyam, V. Labhasetwar, Adv. Drug Deliver. Rev. 2003, 55, 329.

[47] D. W. Hutmacher, Biomaterials 2000, 21, 2529.

[48] W. J. Li, C. T. Laurencin, E. J. Caterson, R. S. Tuan, F. K. Ko, J. Biomed. Mater. Res. 2002, 60, 613.

[49] T. P. Richardson, M. C. Peters, A. B. Ennett, D. J. Mooney, Nat. Biotechnol. 2001, 19, 1029.

[50] J. M. Anderson, M. S. Shive, Adv. Drug Deliver. Rev. 1997, 28, 5.

[51] D. H. Lewis, in Biodegradable Polymers as Drug Delivery Systems, (Eds: M. Chasin, R. Langer), Marchel Dekker, New York 1990, 1.

[52] M. Bognitzki, W. Czado, T. Frese, A. Schaper, M. Hellwig, M. Steinhart, A. Greiner, J. H. Wendorff, Adv. Mater. 2001, 13, 70.

[53] G. Fundueanu, C. Nastruzzi, A. Carpov, J. Desbrieres, M. Rinaudo, Biomaterials 1999, 20, 1427.

[54] K. Gilmore, A. J. Hodgson, B. Luan, C. J. Small, G. G. Wallace, Polym. Gels Networks 1994, 2, 135.

[55] E. R. Kandel, J. H. Schwarts, T. M. Jessel, Principles of Neural Science, Appleton and Lange Norwalk, CT 1991.

[56] K. A. Ludwig, J. D. Uram, J. Y. Yang, D. C. Martin, D. R. Kipke, J. Neural Eng. 2006, 3, 59

[57] A. M. Kuncel, W. M. Grill, Clin. Neurophysiol. 2004, 115, 2431.

[58] D. B. McCreery, W. F. Agnew, T. G. H. Yuen, L. Bullara, IEEE Trans. Biomed. Eng. 1990, 37, 996.

[59] A. Gopferich, Biomaterials 1996, 17, 103.

[60] S. M. Li, H. Garreau, M. Vert, J. Mater. Sci. Mater. Med. 1990, 1, 198.

[61] S. M. Li, H. Garreau, M. Vert, J. Mater. Sci. Mater. Med. 1990, 1, 123.

[62] S. M. Li, S. McCarthy, Biomaterials 1999, 20, 35.

[63] K. Kim, M. Yu, X. H. Zong, J. Chiu, D. F. Fang, Y. S. Seo, B. S. Hsiao, B. Chu, M. Hadjiargyrou, Biomaterials 2003, 24, 4977.

[64] X. H. Zong, S. F. Ran, K. S. Kim, D. F. Fang, B. S. Hsiao, B. Chu, Biomacromolecules 2003, 4, 416.

[65] R. Langer, D. L. Wise, Medical Application of Controlled Release, CRC Press, Boca raton, Florida 2000.

[66] J. A. Tamada, R. Langer, Proc. Natl. Acad. Sci. USA 1993, 90, 552.

[67] F. von Burkersroda, L. Schedl, A. Gopferich, Biomaterials 2002, 23, 4221.

[68] S. Hurrell, R. E. Cameron, Biomaterials 2002, 23, 2401.

[69] M. Dunne, O. I. Corrigan, Z. Ramtoola, Biomaterials 2000, 21, 1659.

[70] R. E. Maxwell, D. M. Long, L. A. French, in Steroids and Brain Edema, (Eds: H. J. Reulen, K. Schihmann), Springer-Verlag, Berlin 1972, 219.

[71] K. E. Uhrich, S. M. Cannizzaro, R. S. Langer, K. M. Shakesheff, Chem. Rev. $1999,99,3181$ 
[72] W. Hoogsteen, A. R. Postema, A. J. Pennings, G. Tenbrinke, P. Zugenmaier, Macromolecules 1990, 23, 634.

[73] S. M. Li, H. Garreau, M. Vert, J. Mater. Sci. Mater. Med. 1990, 1, 131.

[74] A. M. Reed, D. K. Gilding, Polymer 1981, 22, 494.

[75] T. G. Park, S. Cohen, R. Langer, Macromolecules 1992, 25, 116.
[76] R. Arshady, J. Controlled Release 1991, 17, 1.

[77] W. R. Gombotz, S. F. Wee, Adv. Drug Deliver. Rev. 1998, 31, 267.

[78] A. Kikuchi, T. Okano, Adv. Drug Deliver. Rev. 2002, 54, 53.

[79] S. W. Kim, Y. H. Bae, T. Okano, Pharm. Res. 1992, 9, 283. 